\title{
Giant retroperitoneal lumbar Schwannoma: A case report and review of literature
}

\section{Halil Can KÜÇÜKYILDIZ ${ }^{1}$, Salih Kürşat şiMŞEK ${ }^{1}$, Ercan BAL ${ }^{1}$, Serkan ALTINOVA², Murad BAVBEK ${ }^{1}$}

\footnotetext{
${ }^{1}$ Neurosurgery Clinic, Medical Faculty, Ankara Yıldırım Beyazıt University, Ataturk Education and Research Hospital, Ankara, Turkey.

2 Urology Clinic, Ataturk Education and Research Hospital, Ankara, Turkey.
}

\section{SUMMARY}

Schwannomas are benign peripheral nerve sheath tumors arising from Schwann cells. Retroperitoneal localization for schwannomas is extremely rare (\%3). The primary surgical approach for such tumors is total resection. This study aimed to report the case of a schwannoma with giant, retroperitoneal extension originating from the L3 nerve root.

A 43-year-old female patient was admitted to the department with a cellulosic schwannoma pathology after excisional biopsy at another center. The patient's neurological examination was normal. The lumbar-abdominal contrast-enhanced magnetic resonance imaging (MRI) of the patient revealed a mass lesion in the vertebral compartment $2 \times 3 \times 3 \mathrm{~cm}^{3}$ and psoas component $8 \times 4 \times 3 \mathrm{~cm}^{3}$ in size, extending from the right L3 nerve root into the right psoas muscle. The patient was operated postoperatively in the first stage and then using the anterior approach with intraoperative neuromonitoring. Postoperative contrast-enhanced MRI showed no enhancing tumoral lesions. The pathology result was compatible with schwannoma World Health Organization Grade 1.

Two-stage surgical procedure in such cases should be preferred to prove the adequacy of the surgical field and avoid the complications that may occur in neighboring anatomical structures during resection of circumferentially circumscribed tumors.

Urgent surgical intervention is needed for retroperitoneal lumbar schwannomas. Moreover, elective surgery planning is necessary, and preoperative evaluations should be performed.

Key words: Approach, combined surgery, giant schwannoma, lumbar, approach

\section{INTRODUCTION}

Schwannomas are benign peripheral nerve sheath tumors arising from Schwann cells. Spinal schwannomas are generally located in the cervicothoracic junction and lumbosacral component. The thoracic and lumbar localization is rare (1). However, retroperitoneal localization is extremely rare. About 3\% of all schwannomas can be retroperitoneal (2). Some studies have reported the incidence of retroperitoneal schwannoma as $0.7 \%-2.7 \%$ (4). The extraspinal components of the extradural schwannomas are more than $2.5 \mathrm{~cm}$ in size and are called giant spinal schwannomas (3). Giant lumbar schwannomas are rarely reported in the literature. $0.3 \%-5 \%$ of all retroperitoneal tumors are giant paraspinal schwannomas (1).

This paper aimed to report the case of a giant schwannoma with the retroperitoneal extension of the L3 nerve root.

\section{CASE REPORT}

A 43-year-old female patient was admitted to the hospital with a cellulosic schwannoma after excisional biopsy at another center; she complained of right leg pain for 2 years. The neurological examination revealed no weakness and numbness in the lower extremity. Laboratory blood tests also did not show any abnormality.

The lumbar-abdominal contrast-enhanced magnetic resonance Imaging (MRI) of the patient revealed a mass lesion in the vertebral compartment $2 \times 3 \times 3 \mathrm{~cm}^{3}$ and psoas component $8 \times 4 \times 3 \mathrm{~cm}^{3}$ in size, extending from the right L3 nerve 


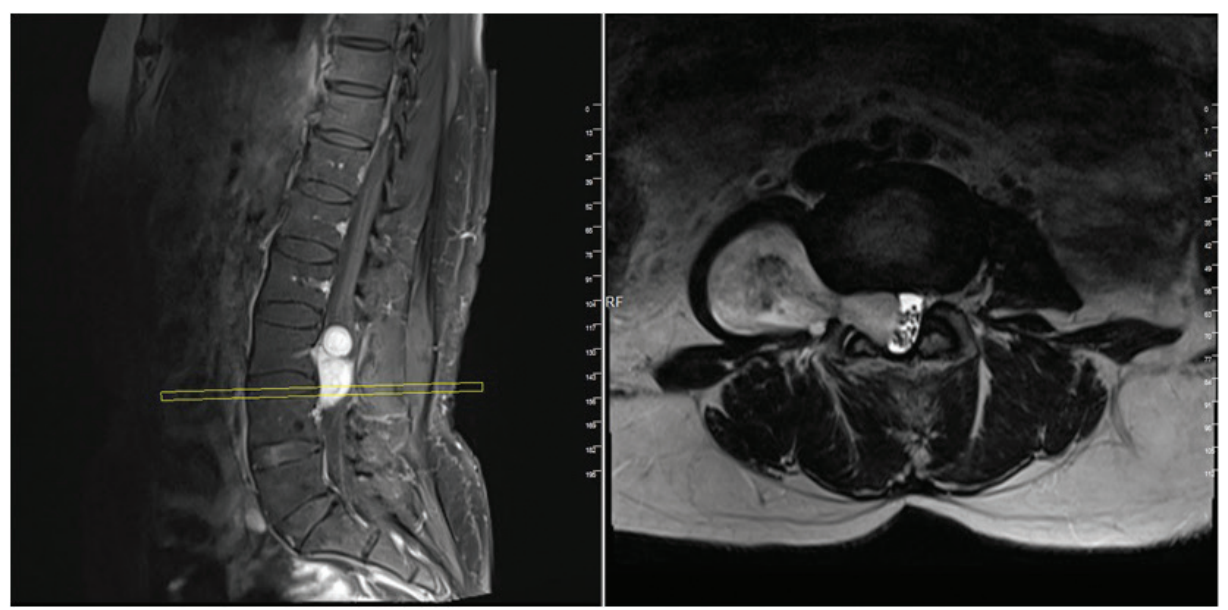

FIGURE 1: Preoperative contrast-enhanced MRI; sagittal/coronal images.

root into the right psoas muscle (Figure 1). The lumbar computed tomography (CT) showed right $\mathrm{L} 3$ neural foramina and restricted bone structure with a destructive appearance.

The patient was operated in the first stage in a prone position in the presence of neuromonitoring. A vertical incision was made from the old incision line. The vertebral column was reached after dissecting the crowns. The limits of $L 2$ and $L 3$ laminectomy performed during the previous excisional biopsy were expanded and the intraspinal component of the tumor was microscopically excised by reaching the right $L 3$ nerve root-related mass. Lumbar instrumentation was not performed because instability was not detected. The flank incision was followed by the anterior retroperitoneal approach to reach the right psoas muscle and the second component of the rest of the body (Figure 2). All the components of the tumor were excised using a two-stage surgical procedure.

No deficits were observed in the postoperative neurological examination. Postoperative contrast-enhanced MRI showed no enhancing tumoral lesions (Figure 3). The end result of the pathology was compatible with the cellular schwannoma World Health Organization Grade 1.

\section{DISCUSSION}

Schwannomas are typically benign, well-limited, encapsulated, slow-growing tumors. Generally, no adhesion to the surrounding tissue is found under the effect of local pressure (5). The surgical operation of schwannomas involves total resection. The peripheral

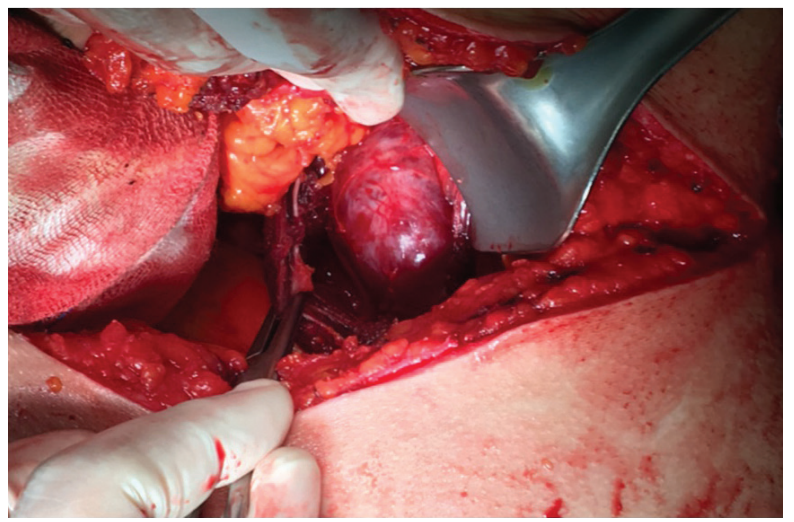

FIGURE 2: Intraoperative appearance of the retroperitoneal component of lumbar schwannoma.

vascular anatomical structures of the tumor should be well assessed in large retroperitoneal schwannomas. This requires careful preoperative neuroradiological evaluation. MRI, CT, or MR angiographic studies in relation to neural tissues and surrounding soft tissue, CT angiographic examination in relation to the vascular component, and $\mathrm{CT}$ images showing osseous structure and destruction of the tumor are useful in the preoperative radiological evaluation of the tumor. Therefore, it is not appropriate to perform a single imaging method in the preoperative evaluation of these tumors. The available literature includes studies reporting preoperative biopsy in selected surveillance of masses that have been extensively retroperitonealized and circumscribed in clinical and radiologic cases (6). Coordination with the relevant surgical team is needed to prevent complications that may occur in intraoperative vascular structures and other retroperitoneal 


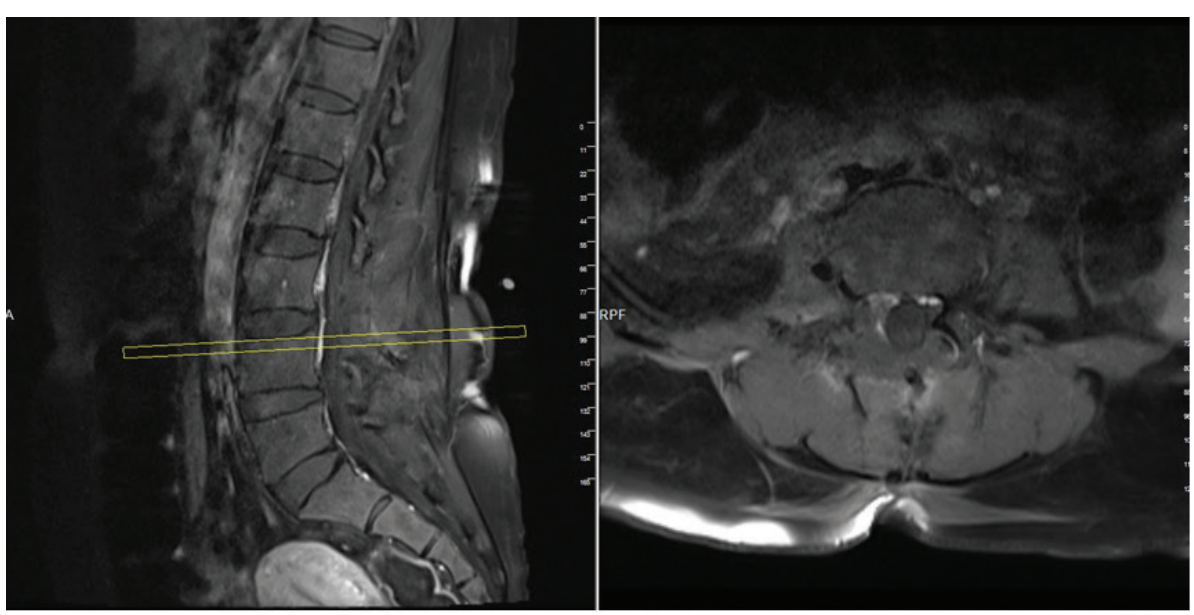

FIGURE 3: Postoperative MRI; no enhancing tumoral lesions.

anatomical structures. Also, the operation should be preferred in the presence of intraoperative neuromonitoring to prevent intrathoracic neural tissue damage.

A schwannoma originates from the spinal nerve sheath. Therefore, exploration of the spinal nerve root associated with the mass is generally possible using the posterior approach followed by exploration and neural decompression after osteotomy in the vertebral column. Once the relationship between the tumor and the nerve root is established, the transperitoneal or retroperitoneal approach should be followed for complete resection of the tumor depending on the extent of the tumor, localization, and extent of distension. However, it may be necessary to apply spinal instrumentation-fusion at the stage of tumor exploration in situations where vertebral column stabilization may be impaired after osteotomy. The surgeon may need to perform one or more surgical procedures depending on the tumor size, localization, and general condition of the patient. In the present case, gross total tumor excision was achieved using the anterior-posterior combined surgical approach. However, Dickson et al., Chang et al., and Sakalauskaite et al. used the two-stage surgical approach (5). It is advocated that the tumor should be removed as much as possible, considering the recurrence rate of the tumor as the main factor in determining the surgical method for the peripheral structures.

The two-stage surgical procedure should be preferred in such cases to prove that the surgical field is adequate and to avoid the complications that may occur in neighboring anatomical structures during resection of these circumferentially circumscribed tumors.
Urgent surgery is needed in such cases because of acute neurological function loss, and various surgical options are available for this purpose. Moreover, elective surgery planning should be clear. Preoperative planning of the surgical approaches provides a better treatment, leading to better clinical outcomes.

\section{REFERENCES}

1. E.-R. Chiang, M.-C. Chang, and T.-H. Chen, "Giant retroperitoneal schwannoma from the fifth lumbar nerve root with vertebral body osteolysis: a case report and literature review," Archives of Orthopaedic and Trauma Surgery, vol. 129, no. 4, pp. 495-499, 2009.

2. Gubbay AD, Moschilla G, Gray BN et al (1995) Retroperitoneal schwannoma: a case series and review. Aust N Z J Surg 65:197-200

3. Surgical management of Giant Lumbar Extradural Schwannoma: Report of 3 cases Seungcheol Lee, MD, Ph.D,1 Umesh Srikantha, M.Ch2 1Department of Neurosurgery, Barunsesang hospital, Bundang-gu, Seongnam-si, Gyeonggido, Republic of Korea, 2Department of Neurosurgery, M S Ramaiah Medical Teaching Hospital, Bangalore, India

4. Q. Li, C. Gao, J. T. Juzi, and X. Hao, "Analysis of 82 cases of retroperitoneal schwannoma," ANZ Journal of Surgery, vol. 77, no. 4, pp. 237-240, 2007.

5. One-Step Posterior and Anterior Combined Approach for L5 Retroperitoneal Schwannoma Eroding a Lumbar Vertebra Giancarlo D'Andrea, Giovanni Sessa, Veronica Picotti, and Antonino Raco S. Andrea Hospital, Institute of Neurosurgery, University of Rome "La Sapienza", Rome, Italy Hindawi Publishing Corporation Case Reports in Surgery Volume 2016.

6. M. Sakalauskaite, J. Stanaitis, S. Cepkus, M. Pleckaitis, and R. Lunevicius, "Retroperitoneal giant schwannoma eroding lumbal vertebra: a case report with a literature review," Central European Journal of Medicine, vol. 3, no. 2, pp. 233-244, 2008. 